\title{
The Future of Infrared; III-Vs or HgCdTe?
}

\author{
MICHAEL A. KINCH ${ }^{1,2,3}$ \\ 1.-DRS Technologies, 13544 N. Central Expressway, Dallas, TX 75243, USA. 2.—e-mail: makinch@ \\ drs-irtech.com.3.-e-mail: mike.kinch@drs.com
}

\begin{abstract}
For reasons associated with size, weight, power consumption, and cost, the future of infrared systems for all spectral bands is being driven towards megapixel formats operating under diffraction- and background-limited conditions with ever-smaller pixel pitches and ever-higher operating temperatures. The performance requirements of such systems with regard to both optical and detector limitations are examined for the materials technologies and device architectures that are in vogue today. At elevated operating temperatures, available noise equivalent temperature difference values for diffraction-limited operation are found to be strongly dependent on the available pixel pitch, optimizing at values $\sim \lambda / 4$, where $\lambda$ is the operating wavelength. The possibility for extending the operation of mid- and longwavelength focal plane arrays to room temperature with diffraction- and background-limited performance is discussed, together with the potential issues that must be addressed in order to achieve this ultimate goal.
\end{abstract}

Key words: $\mathrm{HgCdTe}$, ultra-small pixel, diffraction limit, mid-wave, long-wave, type 2 super-lattice, Shockley-Read lifetime, background limit

\section{INTRODUCTION}

Future infrared (IR) systems will contain megapixel focal plane arrays (FPAs) with ultra-small pitches, and operate at room temperature under diffraction-and background-limited conditions. Operation at the diffraction limit provides maximum resolution and range for the IR system and at the same time limits the maximum sensitivity or minimum available noise equivalent temperature difference (NETD) that can theoretically be achieved. IR system sensitivity can be further compromised by the various attributes of any chosen detector architecture and/or materials technology. It is the purpose of this paper to consider the limitations imposed by the optics and the FPA detector/materials technology with a view to predicting their effect on future system performance at room temperature.

(Received November 18, 2014; accepted February 23, 2015; published online March 12, 2015)

\section{OPTICS LIMITATIONS}

\section{Modulation Transfer Function (MTF)}

The MTF of an optical system with aperture $D$ contains spatial frequencies up to $D / \lambda$, where $\lambda$ represents the operating wavelength of the system. The first zero of the detector MTF for a perfectly delineated FPA with a pixel pitch $d$ occurs at $f / d$, where $f$ is the focal length of the optic. Sampling theory mandates a sampling frequency of at least twice the highest frequency contained in the optics MTF to achieve an uncompromised image. Thus $f /$ $d>2 D / \lambda$, or alternatively $F \lambda / d>2$, where $F$ represents the $F / \#$ of the optic. The individual MTF components associated with an optical system of aperture $D$, with a focal length $f$, and a perfectly defined detector pixel with pitch $d$ are shown in Fig. 1 for an arbitrary wavelength $\lambda$, together with the product of those components for the condition $F \lambda / d=2$. The combined MTF is seen to be virtually identical to that of the optics alone, indicating that the condition $F \lambda / d>2$ represents an optimum condition for optical system design, ${ }^{1}$ ensuring diffraction-limited performance with a minimum of over-sampling. Systems with slower optics can be utilized, but will provide no improvement in system 


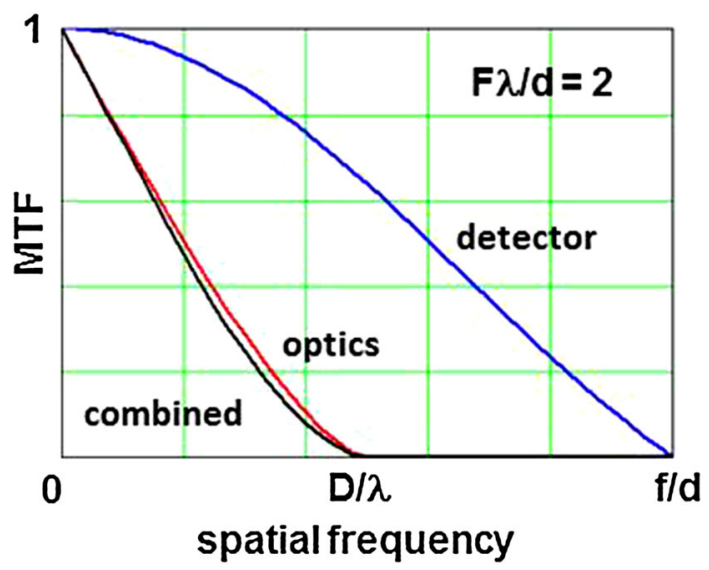

Fig. 1. MTF versus spatial frequency for an optic aperture $D$ with focal length $f$ and a detector of pitch $d$ operating at a wavelength $\lambda$ for the condition $F / / d=2$.

resolution, and in fact will be seen to result in reduced system sensitivity.

\section{Noise Equivalent Temperature Difference (NETD)}

The NETD of an FPA is given by

$$
\mathrm{NETD}=\frac{\left[1+\left(J_{\mathrm{d}} / J_{\Phi}\right]\right.}{\eta_{\mathrm{CS}} C \sqrt{\left(J_{\mathrm{d}}+J_{\Phi}\right) A \tau_{\mathrm{int}} / q}},
$$

where $J_{\mathrm{d}}$ is detector dark current, $J_{\Phi}$ the total background flux current density, $C=\left[\left(\mathrm{d} \Phi_{\mathrm{B}} / \mathrm{d} T\right) / \Phi_{\mathrm{B}}\right]$ is the scene contrast through the optics, $A$ is the pixel area, $\tau_{\text {int }}$ the integration time, and the cold shield efficiency is given by

$$
\eta_{\mathrm{CS}}=\left[\Phi_{\mathrm{B}}^{\mathrm{optics}} / \Phi_{\mathrm{B}}^{\mathrm{total}}\right]
$$

At low temperatures, $J_{\mathrm{d}}=0$, and $\eta_{\mathrm{CS}}=1$. For $F>1$, we then have

$$
\begin{aligned}
\mathrm{NETD}= & \frac{\left[1+\left(J_{\mathrm{d}} / J_{\Phi}\right)\right]}{\eta_{\mathrm{CS}} C \sqrt{\left(J_{\mathrm{d}}+J_{\Phi}\right) A \tau_{\mathrm{int}} / q}} \approx \frac{\sqrt{1+4 F^{2}}}{C d \sqrt{\eta_{\mathrm{CS}} \Phi_{\mathrm{B}}^{2 \pi} \tau_{\mathrm{int}}}} \\
& \approx \frac{2}{C \lambda \sqrt{\Phi_{\mathrm{B}}^{2 \pi} \tau_{\mathrm{int}}}} \times\left[\frac{F \lambda}{d}\right] .
\end{aligned}
$$

Thus, at low temperatures, the minimum available NETD in the diffraction limit, as given by Eq. 3, is pre-determined to within an integration time, which is in turn limited by the system frame rate. The minimum available NETD under the condition $F \lambda$ / $d=2$, together with the required unit cell well capacity, is shown in Fig. 2 as a function of cutoff wavelength for a $30-\mathrm{Hz}$ system looking at a $300 \mathrm{~K}$ scene.

In the diffraction limit, it is readily apparent that the performance at low operating temperatures predicted by Eq. 3 is independent of pixel size. In

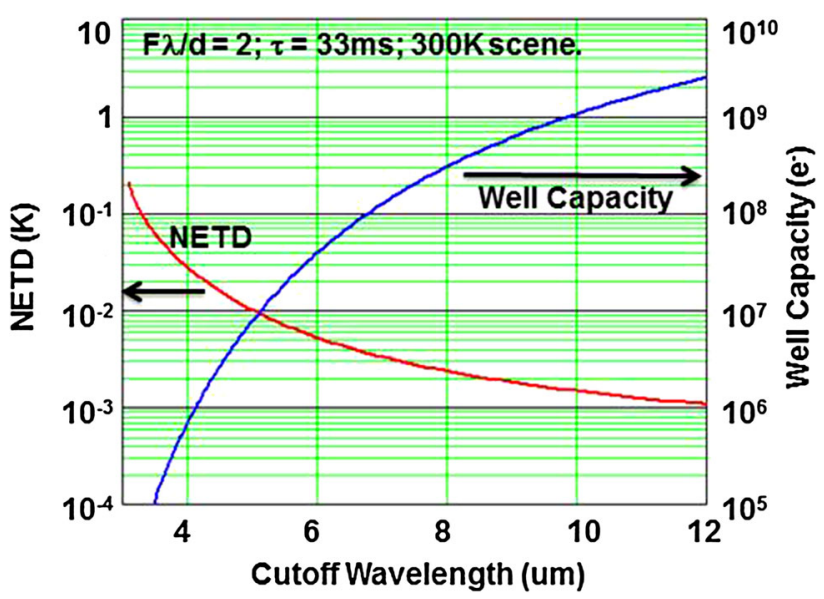

Fig. 2. Minimum available low temperature NETD and required well capacity as a function of cutoff wavelength for a diffraction-limited $30 \mathrm{~Hz}$ optical system with $F \lambda / d=2$.

these circumstances, the driving force to reduce the pixel pitch is purely one of a reduction in the size, weight, power, and cost of the IR system. However, as one increases the operating temperature, then minimizing the pixel pitch takes on another dimension, namely one involving system performance.

Consider the effect of the condition, $F \lambda / d=2$, on the cold shield efficiency of the detector as the temperature is elevated. The cold shield efficiency is defined by

$$
\eta_{\mathrm{CS}}=\Phi_{\mathrm{B}}^{\text {optics }} / \Phi_{\mathrm{B}}^{\text {total }}=\Phi_{\mathrm{B}}^{\text {optics }} /\left[\Phi_{\mathrm{B}}^{\text {optics }}+\Phi_{\mathrm{B}}^{\mathrm{CS}}\right]
$$

and, as the temperature is raised, then $\Phi_{\mathrm{B}}^{\mathrm{CS}}>\Phi_{\mathrm{B}}^{\text {optics }}$, and $\eta_{\mathrm{CS}}$ becomes $<1$, and depends on pixel pitch in the diffraction-limited condition.

The NETD of the detector for $F \lambda / d=2$, again neglecting dark current, is now given by

$$
\begin{aligned}
\mathrm{NETD} & =\frac{\left[1+\left(J_{\mathrm{d}} / J_{\Phi}\right)\right]}{\eta_{\mathrm{CS}} C \sqrt{\left(J_{\mathrm{d}}+J_{\Phi}\right) A \tau_{\mathrm{int}} / q}}=\frac{\sqrt{\left(1+4 F^{2}\right)}}{C d \sqrt{\eta_{\mathrm{CS}} \Phi_{\mathrm{B}}^{2 \pi} \tau_{\mathrm{int}}}} \\
& \approx \frac{4}{C \lambda \sqrt{\eta_{\mathrm{CS}} \Phi_{\mathrm{B}}^{2 \pi} \tau_{\mathrm{int}}}} .
\end{aligned}
$$

The NETD now depends on $\eta_{\mathrm{CS}}$, and hence pixel size.

It is now meaningful to model system NETD performance as a function of operating temperature according to Eqs. 4 and 5 for various pixel pitches. This is shown in Fig. 3a for the specific case of a mid-wave (MW) FPA with pixel pitches of $20 \mu \mathrm{m}, 10$ $\mu \mathrm{m}, 5 \mu \mathrm{m}$ and $2.5 \mu \mathrm{m}$. The minimum available NETD for a $30-\mathrm{Hz}$ system is seen to be strongly dependent on pixel pitch at temperatures $>180 \mathrm{~K}$, due simply to the dependence of the cold shield efficiency on pixel pitch, which is shown in Fig. $3 \mathrm{~b}$ 


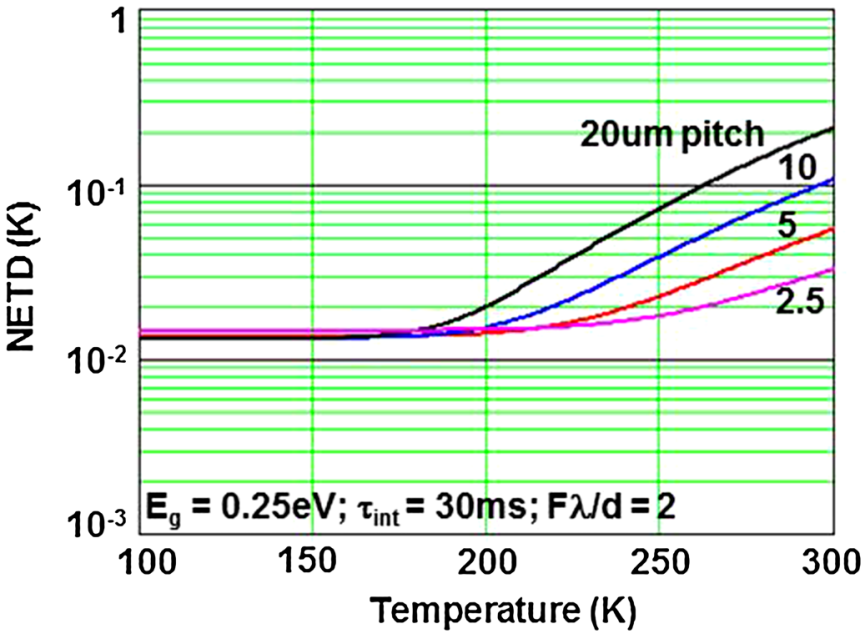

(a)

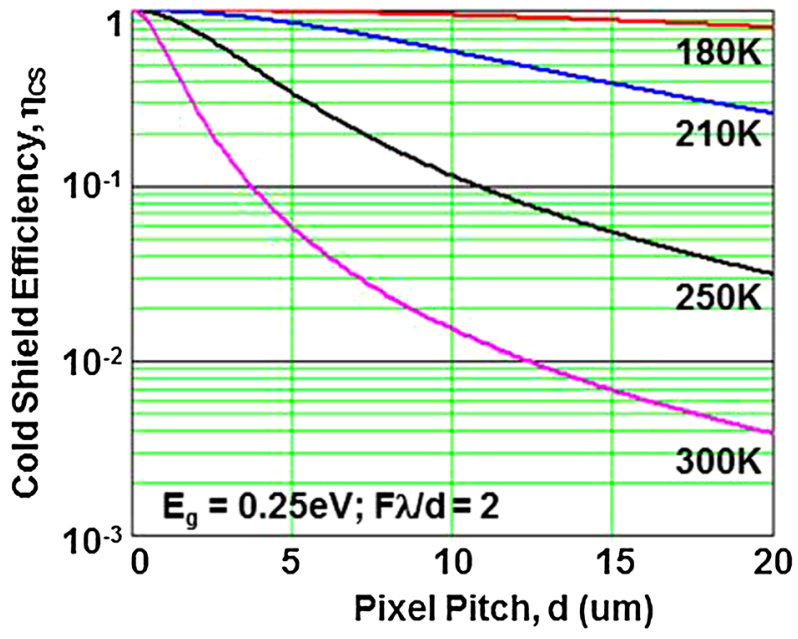

(b)

Fig. 3. (a) MWIR NETD versus operating temperature for various pixel pitches and (b) cold shield efficiency versus pixel pitch at various operating temperatures for $F \lambda / d=2$ and a $30-\mathrm{Hz}$ system.

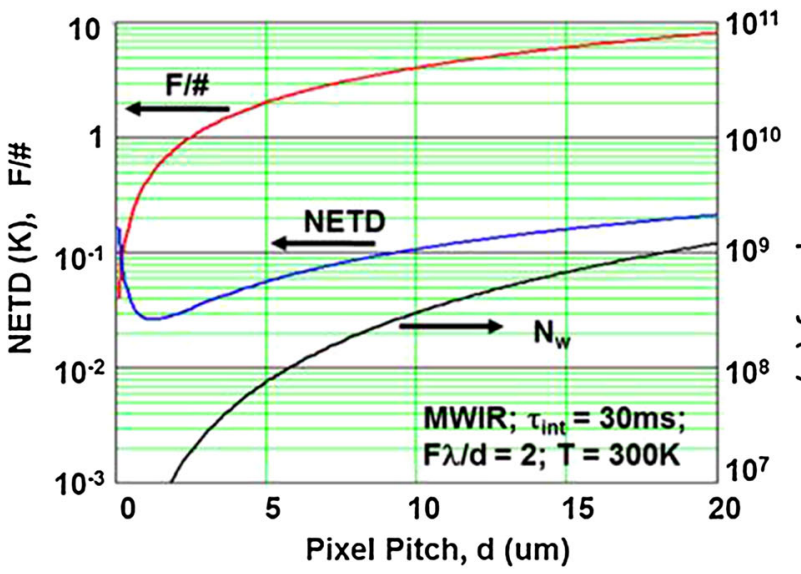

(a)

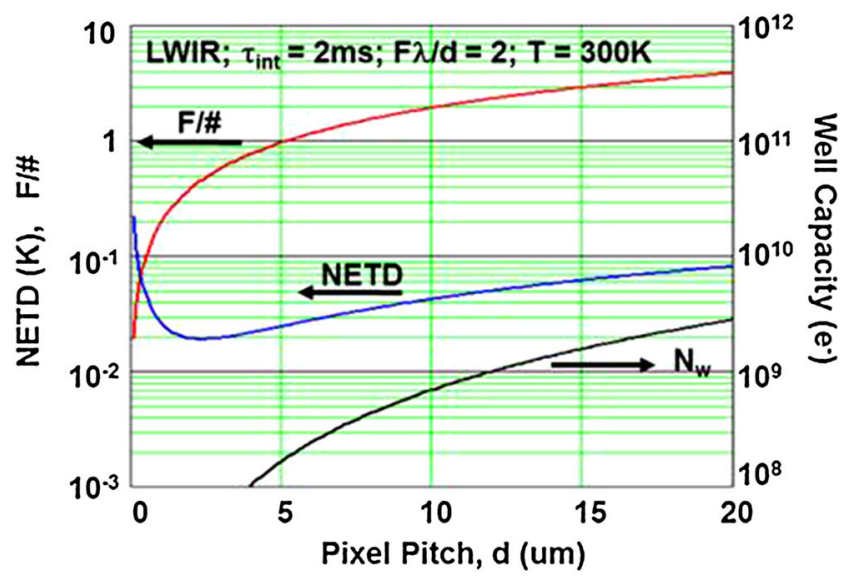

(b)

Fig. 4. Available NETD, required well capacity, and F/\# versus pixel pitch for (a) 30-Hz MWIR and (b) 500-Hz LWIR FPAs operating at the diffraction limit at $300 \mathrm{~K}$.

for various operating temperatures. The minimum NETD at $300 \mathrm{~K}$ for a $2.5-\mu \mathrm{m}$ pitch is seen to approach $30 \mathrm{mK}$. Larger pitches for a MWIR FPA result in larger NETD values at $300 \mathrm{~K}$ in the diffraction limit. Lower MWIR NETD values can then only be achieved with faster optics, and hence poorer resolution.

The diffraction-limited NETD at an operating temperature of $300 \mathrm{~K}$ as a function of pixel pitch is shown in Fig. 4a for a 30-Hz MWIR system, together with the required well capacity and system $F / \#$. The NETD is seen to minimize at $d \sim \lambda / 4$, when $F \sim 0.5$, as indicated by Eq. 5. Similar arguments apply to diffraction-limited LWIR FPAs, as illustrated in Fig. 4b for a 500-Hz system. The availability of more flux in the LW spectral band is seen to enable lower
NETD values at relatively short integration times, but at the expense of storing more charge.

\section{DETECTOR LIMITATIONS}

Limitations imposed on the IR system by the detector/readout integrated circuit (ROIC) are:

- Dark current

- Pixel delineation

- Pixel hybridization

- ROIC well capacity

\section{Dark Current}

To estimate the impact of dark current on possible room temperature background-limited FPA 
operation, we must first assess the various device architectures and materials technologies in vogue today for the MW and LW spectral bands. There are available three detector architectures and two potential materials technologies. The detector architectures are:

1. The photodiode

2. The barrier layer photoconductor

3. The PIN photodiode.

The photodiode can be implemented in either a double layer hetero-junction (DLHJ) or a homojunction format, and its dark current is bi-polar and will typically exhibit both diffusion and depletion components. The barrier layer photoconductor on the other hand is a unipolar minority carrier device, and possesses the somewhat unique capability of flat-band absorber operation if desired. This property is particularly useful for materials technologies with a short Shockley-Read (S-R) lifetime. The absorber can be a uniform alloy, or an engineered bandgap material such as a type 2 super-lattice (T2SL). The PIN architecture is merely a low-doped photodiode that is biased into complete depletion, thus generating an intrinsic absorber volume from which diffusion currents are absent.

The materials technologies that can potentially impact diffraction-limited FPA operation at room temperature are:

1. III-Vs.

2. HgCdTe ternary alloys.

III-V IR materials consist of appropriate compositions of the $k=0$ direct bandgap ternary alloy InAsSb, or bandgap engineered T2SLs consisting of alternating layers of $\operatorname{InAs} / \mathrm{GaSb}$, or InAs/InAsSb. The $\mathrm{S}-\mathrm{R}$ lifetime of the Sb-like alloys is $<1 \mu \mathrm{s}$, which mandates the use of the barrier layer photoconductor architecture for operation at elevated temperatures for both the alloy and the T2SL. The T2SL offers the advantage of Auger suppression by a suitable reduction of the phase space available for Auger transitions. For reasons of surface passivation and tolerance to threading dislocations, the preferred format is $\mathrm{nBn}$. Doping concentrations are typically limited to values $>10^{15} / \mathrm{cm}^{3}$, for $N$-type and $P$-type, with the exception of InSb, which is available with $N \sim 10^{14} / \mathrm{cm}^{3}$. HgCdTe is a $k=0$ direct bandgap ternary alloy with a composition that is appropriate for the spectral band in question. S-R lifetime values are $>100 \mu \mathrm{s}$ for $\mathrm{LW}$, and $>10 \mathrm{~ms}$ for MW, and consequently HgCdTe is compatible with all three of the above detector architectures. Available doping concentrations are $>10^{14} / \mathrm{cm}^{3}$ for both $N$-type and $P$-type, ${ }^{2}$ although low values of $P$-type doping are not typically utilized in $\mathrm{HgCdTe}$ architectures due to the extreme sensitivity of the material to donor surface charge and donor core dislocations.

It is meaningful to model the available dark currents as a function of temperature for the above MW and LW materials. The various dark current components are given $b^{3}$ :

Auger diffusion current:

$$
J_{\mathrm{A}}=\frac{q N_{\mathrm{maj}} t}{2 \tau_{\mathrm{Ai}}}
$$

S-R diffusion current:

$$
J_{\mathrm{SR}}=\frac{q n_{i}^{2} t}{\left(N_{\mathrm{maj}}+2 n_{i}\right) \tau_{\mathrm{SR}}}
$$

S-R depletion current:

$$
J_{\mathrm{dep}}=\frac{q n_{i} t}{2 \tau_{\mathrm{SR}}}
$$

where $N_{\text {maj }}$ represents the appropriate majority carrier concentration, $t$ is the absorber thickness, $\tau_{\mathrm{Ai}}$ the appropriate intrinsic Auger lifetime, and $\tau_{\mathrm{SR}}$ the S-R lifetime.

For MWIR (constant $E_{\mathrm{g}}=0.25 \mathrm{eV}$ ) the modeled dark currents as a function of temperature utilizing Eqs. 6 through 8 are shown in Fig. 5 for the flatband $\mathrm{nBnInAsSb}$ alloy absorber, the $\mathrm{HgCdTeP}^{+} / \mathrm{N}$ DLHJ, the $\mathrm{HgCdTeN}^{+} / \mathrm{P}$ DLHJ, and the $\mathrm{HgCdTe}$ PIN diode. The S-R lifetime for InAsSb is chosen to be $400 \mathrm{~ns},{ }^{4,5}$ and for HgCdTe is $4 \mathrm{~ms} .{ }^{6,7}$

A similar model for constant $0.12 \mathrm{eV}$ LWIR materials is shown in Fig. 6 except that the barrier layer photoconductor utilizes an Auger-suppressed $\mathrm{pBpInAs} / \mathrm{GaSb}$ T2SL with a lifetime of $40 \mathrm{~ns}$. Use of an InAs/InAsSb T2SL would provide a lifetime of $\sim 400 \mathrm{~ns}$ and reduce the dark current by a factor of 10 for this architecture.

At any given temperature, Eqs. 6 and 7 indicate that if both Auger and S-R diffusion components are present then the dark current will optimize at a specific doping level. This is illustrated in Fig. 7 for MWIR absorbers at two specific operating temperatures, namely $150 \mathrm{~K}$ and $300 \mathrm{~K}$. The operating conditions of the four technologies considered in Fig. 5 are indicated, together with the appropriate background flux for diffraction-limited operation. It is apparent that all four materials architectures meet the requirements for background limited operation (BLIP) at $150 \mathrm{~K}$, but only the $\mathrm{HgCdTe} \mathrm{PIN}$ diode is BLIP at $300 \mathrm{~K}$, due solely to the presence of Auger diffusion current in the other technologies. Auger generation can be suppressed by the use of a T2SL absorber for the $\mathrm{nBn}$ architecture, but the BLIP requirement on S-R lifetime for $N=10^{16} / \mathrm{cm}^{3}$ is seen to be $>200 \mu \mathrm{s}$. Current state of the art for III-V materials is $<1 \mu \mathrm{s} ., 5$

Similar arguments can be made for LWIR, as shown in Fig. 8, for operating temperatures of $120 \mathrm{~K}$ and $300 \mathrm{~K}$. In this case, the required S-R lifetime is $>100 \mu \mathrm{s}$ for both the Auger-suppressed T2SL and the PIN diode. 

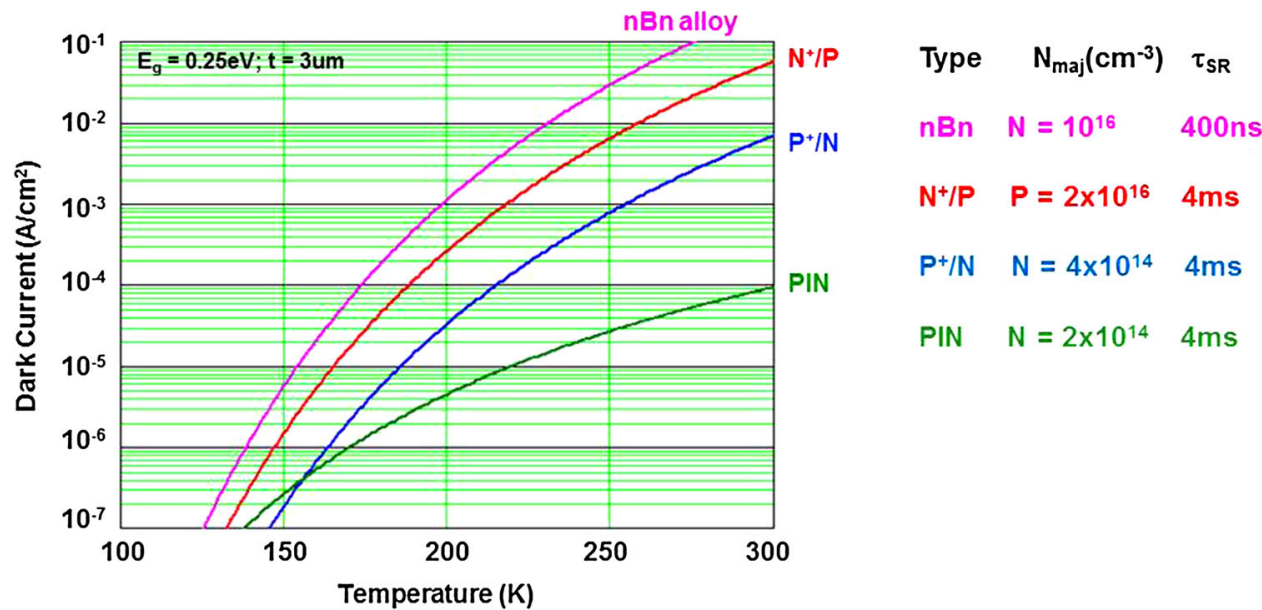

merature $(\mathrm{K})$

Fig. 5. Model of dark current versus temperature for $0.25 \mathrm{eV}$ bandgap nBnlnAsSb alloy, $\mathrm{N}^{+} / \mathrm{P} \mathrm{HgCdTe}, \mathrm{P}^{+} / \mathrm{N} \mathrm{HgCdTe}$, and $\mathrm{PIN} \mathrm{HgCdTe}$ absorbers with the indicated materials properties.

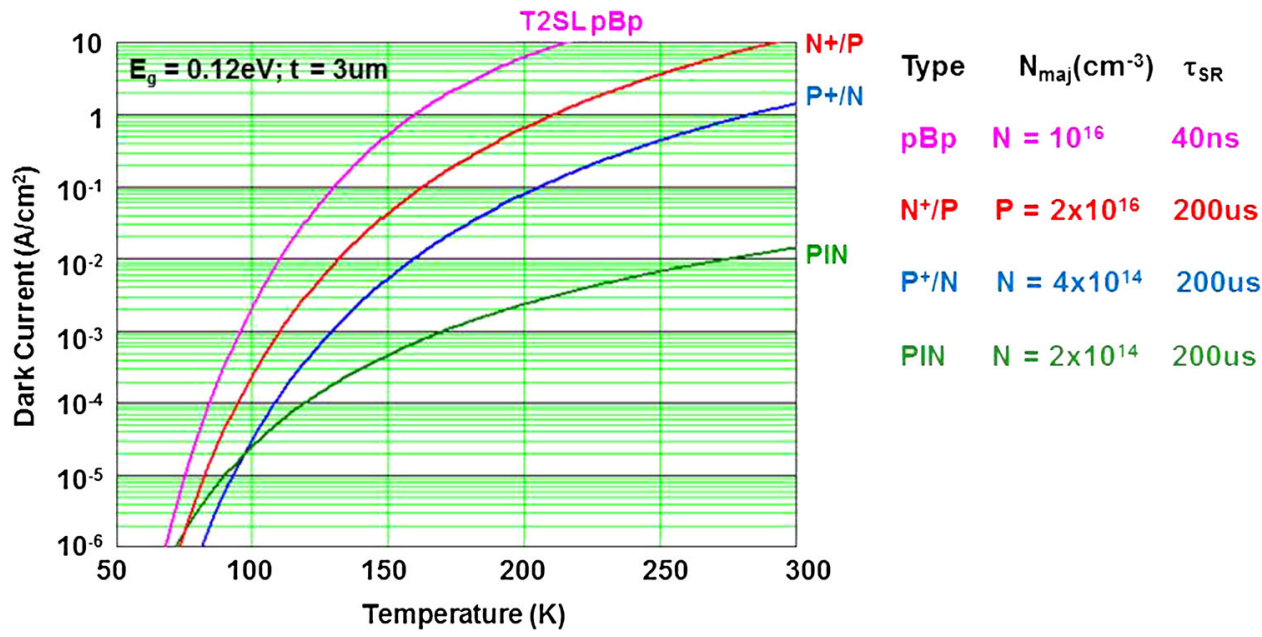

Fig. 6. Model of dark current versus temperature for $0.12 \mathrm{eV}$ bandgap pBplnAs/GaSb T2SL, $\underline{\mathrm{N}}^{+} / \mathrm{P} \mathrm{HgCdTe}, \underline{\mathrm{P}}^{+} / \mathrm{N} \mathrm{HgCdTe}$ and PIN HgCdTe absorbers with the indicated materials properties.

We are thus confronted with two options for achieving MWIR and LWIR BLIP performance under diffraction limited operating conditions at room temperature, namely:

1. The diffusion-limited $\mathrm{nBn}$ T2SL barrier layer photoconductor with $N>10^{16} / \mathrm{cm}^{3}$, and $\tau_{\mathrm{SR}}>$ $200 \mu$ s for MWIR, and $>100 \mu \mathrm{s}$ for LWIR.

2. The depletion-limited PIN photodiode with $\tau_{\mathrm{SR}}>$ $1 \mathrm{~ms}$ for MWIR, and $>100 \mu \mathrm{s}$ for LWIR.

The projected MWIR NETD performance as a function of temperature for these two materials architectures can be modeled by incorporating the dark currents contained in Eqs. 6 through 8 into the full-up NETD expression defined by Eq. 5 for the various values of pixel pitch, S-R lifetime and available well capacity indicated in Table I. This dependence is shown in Fig. 9a for the Auger-suppressed InAs/InAsSb T2SL architecture assuming an absorber thickness of $3 \mu \mathrm{m}$, a collection efficiency of 0.6 , and a $30-\mathrm{Hz}$ frame rate. For III-V S-R lifetime values of $<1 \mu \mathrm{s}$, it is apparent that background flux limited performance (BLIP) at the diffraction limit at room temperature is not currently possible for this materials architecture. An S-R lifetime of $>200 \mu \mathrm{s}$ is required to achieve such a limit in MWIR III-V material at $300 \mathrm{~K}$.

The modeled NETD performance as a function of temperature of the MWIR $\mathrm{HgCdTeP}^{+} / \mathrm{N}$ DLHJ is shown in Fig. 9b, assuming the currently available HgCdTe parameter values shown in Table I. Again it is apparent that diffusion limited operation with this materials architecture will not enable BLIP operation at room temperature, and the PIN architecture must be employed in order to eliminate the Auger dark current component. This mode of operation requires relatively low doping concentrations of $<2 \times 10^{14} / \mathrm{cm}^{3}$, so as to completely deplete the absorber at modest values of reverse bias $(<1 \mathrm{~V})$, but does enable optics limited performance 


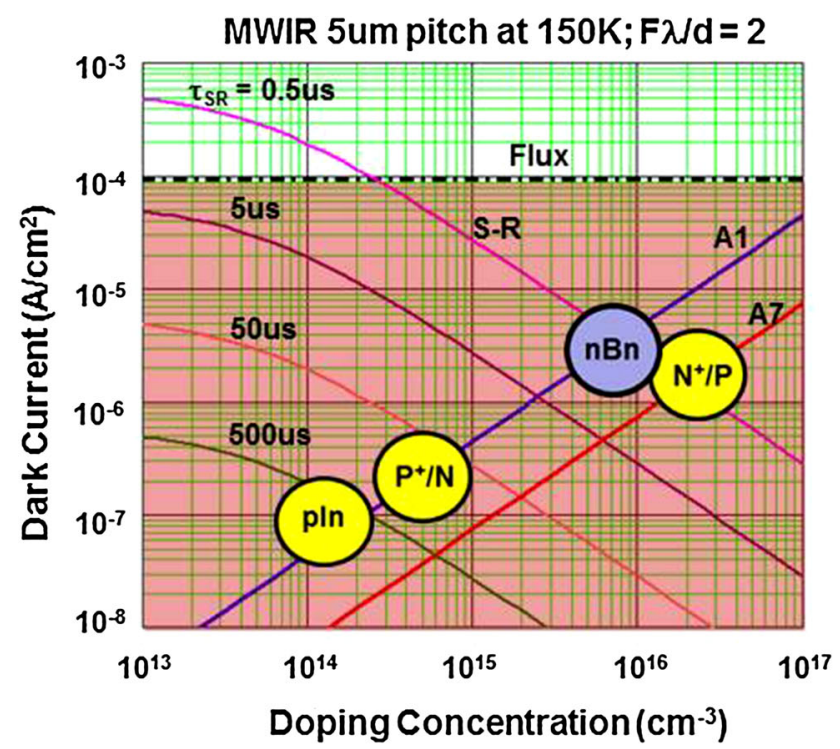

(a)

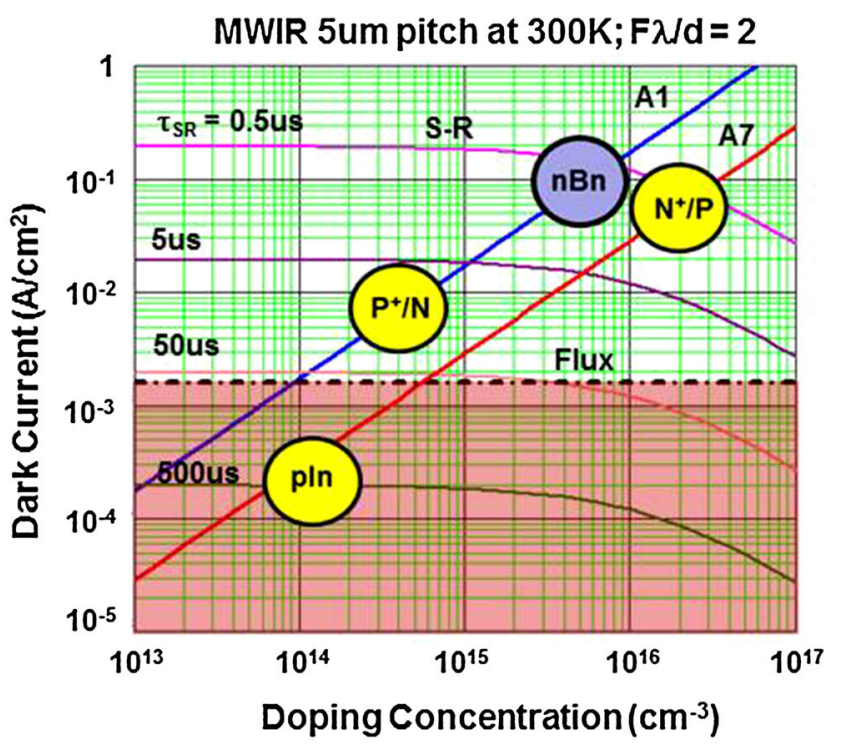

(b)

Fig. 7. Auger and S-R diffusion currents versus majority carrier doping concentration for MWIR architectures at (a) $150 \mathrm{~K}$, and (b) $300 \mathrm{~K}$.

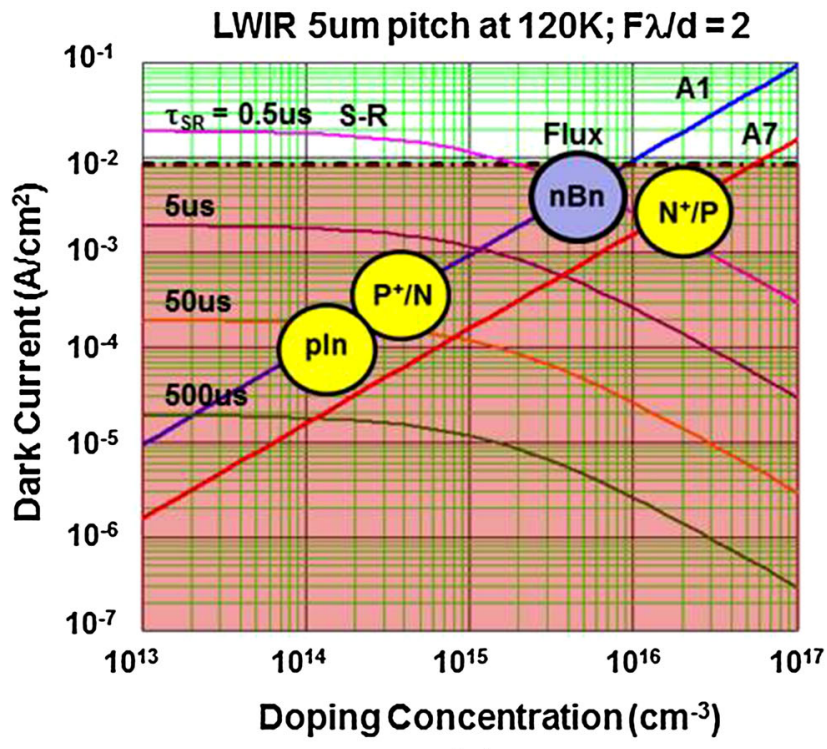

(a)

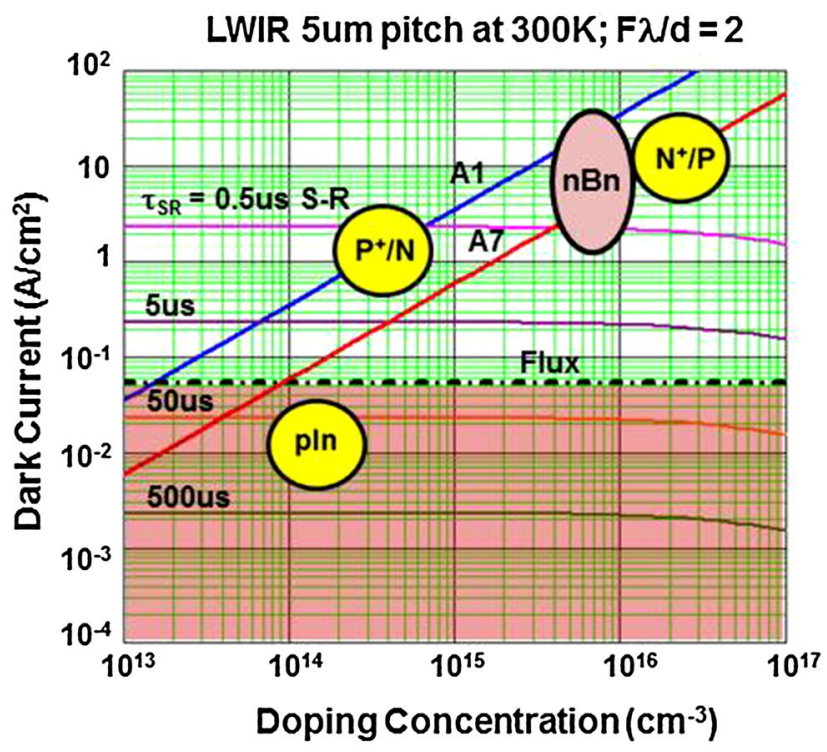

(b)

Fig. 8. Model of Auger and S-R diffusion currents versus majority carrier doping concentration for LWIR materials at (a) $150 \mathrm{~K}$, and (b) $300 \mathrm{~K}$.

at room temperature using currently available HgCdTe.

Again, it is emphasized that optics-limited performance at the diffraction limit is limited to NETD values $\sim 60 \mathrm{mK}$ for the minimum pitch of $5 \mu \mathrm{m}$ assumed in Table I. To achieve a desired value of $30 \mathrm{mK}$ at room temperature under diffraction limited conditions requires the use of a $2.5 \mu \mathrm{m}$ pixel pitch as was pointed out earlier.

Similar arguments can be made for LWIR NETD performance versus operating temperature. In this case photon fluxes are considerably higher, and as such faster frame rates can be used whilst still achieving BLIP performance, but at the expense of larger required well capacities to store the integrated charge.

\section{Pixel Delineation}

The MTF requirements of ultra-small pitches with relatively low dark currents mandate the almost complete delineation of the individual pixels in an FPA. This can be achieved in one of two ways, namely: 
Table I. Material/architecture parameters for the InAs/InAsSb T2SL and HgCdTe diode models

\begin{tabular}{|c|c|c|c|c|c|}
\hline Material/architecture & $N_{\text {maj }}\left(\mathrm{cm}^{-3}\right)$ & $\tau_{\mathbf{S R}}(\mu \mathbf{S})$ & $\operatorname{Pitch}(\mu \mathrm{m})$ & Well capacity $\left(\mathbf{e}^{-}\right)$ & $F \lambda / d$ \\
\hline \multicolumn{6}{|l|}{ III-IV } \\
\hline 1. T2SL & $10^{16}$ & 1 & 15 & $3 \times 10^{6}$ & 2 \\
\hline 2. T2SL & $10^{16}$ & 1 & 5 & $3 \times 10^{6}$ & 2 \\
\hline 3. T2SL & $10^{16}$ & 1 & 5 & $10^{10}$ & 2 \\
\hline 4. T2SL & $10^{16}$ & 200 & 5 & $10^{8}$ & 2 \\
\hline \multicolumn{6}{|l|}{ HgCdTe } \\
\hline 1. $\mathrm{P}^{+} / \mathrm{N} \mathrm{HJ}$ & $4 \times 10^{14}$ & 4000 & 15 & $3 \times 10^{6}$ & 2 \\
\hline 2. $\mathrm{P}^{+} / \mathrm{N}$ HJ & $4 \times 10^{14}$ & 4000 & 5 & $3 \times 10^{6}$ & 2 \\
\hline 3. $\mathrm{P}^{+} / \mathrm{N}$ HJ & $4 \times 10^{14}$ & 4000 & 5 & $10^{9}$ & 2 \\
\hline 4. PIN & $2 \times 10^{14}$ & 4000 & 5 & $10^{8}$ & 2 \\
\hline
\end{tabular}

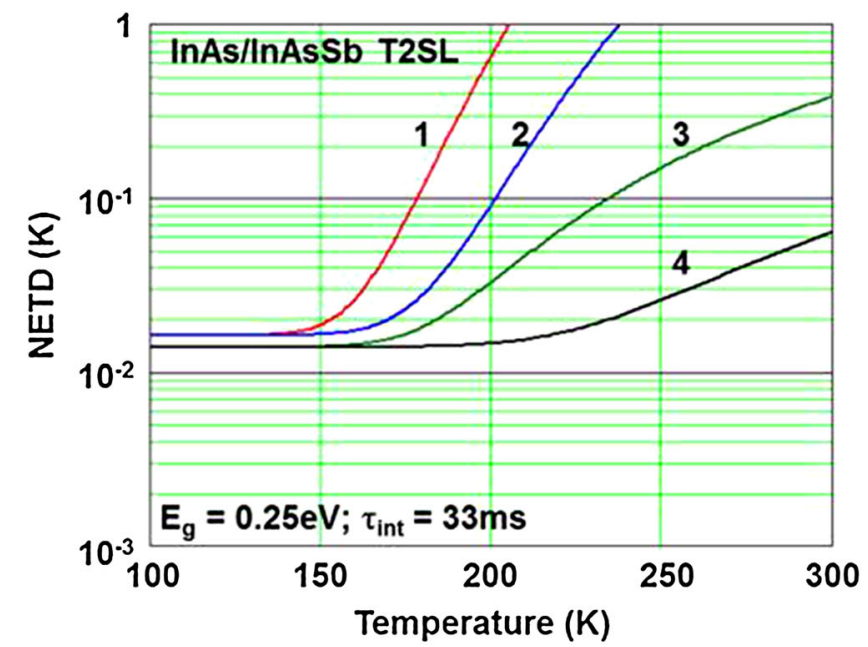

(a)

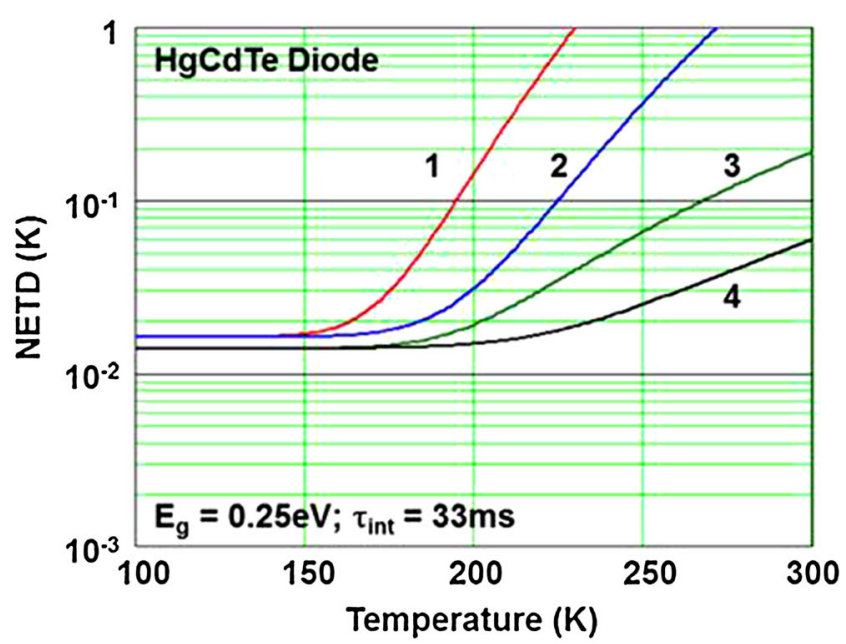

(b)

Fig. 9. MWIR NETD versus temperature for various (a) Auger-suppressed T2SL, and (b) HgCdTe diode absorbers in the diffraction limit for the parameters listed in Table I.

1. Physically, by the etching of mesas, and

2. Electrically, by the use of built-in electric fields.

The diffusion-limited dark current option discussed above requires mesa etching. This is relatively straightforward except for the limitations imposed by the requirement of almost completely etching through an absorption thickness $(\sim 3 \mu \mathrm{m})$ of material, followed by a subsequent surface passivation process, all within the confines of the ultra-small pitch geometry.

Electrical delineation of a $5-\mu \mathrm{m}$ pitch with excellent MTF has already been demonstrated on the DARPA Lambda Scale Program ${ }^{8}$ utilizing the cylindrical diode geometry of the high density vertically integrated photodiode (HDVIP) architecture, but this architecture is Auger diffusion currentlimited, which eliminates it from consideration as a BLIP room temperature photon detector. However, the depletion current-limited PIN diode can realistically satisfy the MTF requirement associated with ultra-small pitches.

\section{Pixel Hybridization}

Hybridization of the FPA to the silicon ROIC can be accomplished by metal bumps or by etched vias through the IR material. The only option realistically available for the two architectures proposed for potential room temperature BLIP operation is the metal bump bond, which, however, has yet to be demonstrated for the ultra-small pitches required for diffraction-limited performance.

\section{ROIC Well Capacity}

BLIP operation of a photon detector at room temperature requires that the charge from the full $2 \pi$ field of view that is integrated during a frame time be effectively stored on the detector node, even as the pixel pitch is being decreased. This storage requirement is illustrated in Fig. 10 for a $5-\mu \mathrm{m}$ detector pixel as a function of system cutoff wavelength, indicating that a significantly larger well capacity per $\mu \mathrm{m}^{2}$ is required to achieve BLIP 


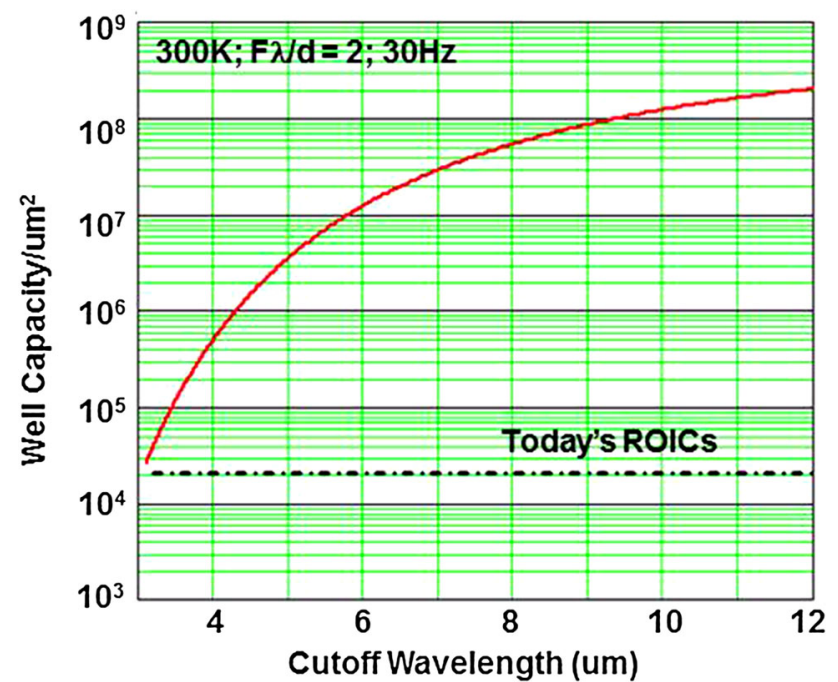

Fig. 10. Required well capacity $/ \mu \mathrm{m}^{2}$ as a function of cutoff wavelength for BLIP operation at room temperature in the diffraction limit for a $30-\mathrm{Hz}$ frame rate.

performance at room temperature compared to the values available with current ROIC designs. It is readily apparent that a paradigm shift is required in ROIC design to achieve the desired goal.

\section{CONCLUSIONS}

Diffraction-limited resolution without image aliasing for any IR system is achieved for the condition $F \lambda / d=2$. At low temperatures, where the detector cold shield efficiency $\eta_{\mathrm{CS}} \sim 1$, both the system resolution and NETD are determined solely by the $(F \lambda / d)$ parameter for a given frame rate. Optimum performance is independent of pixel pitch if the condition $F \lambda / d=2$ is maintained. Thus, at low temperatures, the driving force for ultra-small pixels is purely and simply the enabled reduction in system size, weight, power and cost. However, at high temperatures, where cold shield efficiency is a relevant parameter, a further benefit is gained from the use of ultra-small pixels, namely improved NETD performance, with optimum performance achieved for a pixel pitch of $\lambda / 4$ at $F \sim 0.5$.

Our analysis shows that BLIP performance is possible at room temperature for both the MW and LW spectral bands provided that Auger dark current is suppressed in the IR absorber, and a suitably long S-R lifetime exists This can theoretically be achieved either by the InAs/InAsSb T2SL architecture or the HgCdTe PIN diode. The required S-R lifetime depends on the architecture, but values $>100 \mu$ s are necessary for LW, and $>200$ to $1000 \mu \mathrm{s}$ for MW. HgCdTe is currently the only materials technology that satisfies these requirements. III-V alloys typically exhibit S-R lifetimes $<1 \mu \mathrm{s}$.

The implementation of ultra-small pixel room temperature FPAs will require significant technology development in the areas of (1) pixel delineation, (2) ROIC hybridization, and (3) enhanced ROIC well capacity density.

\section{ACKNOWLEDGEMENT}

The author wishes to acknowledge one of the referees for drawing his attention to a mathematical error.

\section{REFERENCES}

1. G.C. Holst and R.G. Driggers, Opt. Eng. 51, 096401 (2012).

2. D. Chandra, D.F. Weirauch, H.F. Schaake, M.A. Kinch, F. Aqariden, C.F. Wan, and H.D. Shih, J. Electron. Mater. 34, 963 (2005).

3. M.A. Kinch, State-of-the-Art Infrared Detector Technology (Bellingham: SPIE Press, 2014), p. 48.

4. S.R. Jost, V.F. Meikleham, and T.H. Myers, MRS Sympos 90, 429 (1987).

5. E.H. Steenbergen, B.C. Connelly, G.D. Metcalfe, H. Shen, M. Wraback, D. Lubyshev, Y. Qiu, J.M. Fastenau, A.W.K. Liu, S. Elhamri, O.O. Cellek, and Y.-H. Zhang, Appl Phys Lett 99, 251110 (2011).

6. J. Pellegrino, R. DeWames, P. Perconti, C. Billman, and P. Maloney, Proc. SPIE 8353, 83532X (2012).

7. DRS internal data.

8. J.M. Armstrong, M.R. Skokan, M.A. Kinch, and J.D. Luttmer, Proc. SPIE 9070, 907033-1 (2014). 